\title{
AN APPLICATION OF THE MAURO PICONE THEOREM FOR HEAT CONDUCTION ${ }^{1}$
}

\author{
GEORGE W. EVANS II $^{2}$
}

For the application presented in this paper we shall use the following restricted statement of a theorem proved by Mauro Picone. ${ }^{1}$

THEOREM. Let $R$ be a simply connected region in the xt-plane, where $0 \leqq t \leqq T$, with a part of the boundary of $R$ being $t=T$ and the remaining part of the boundary being given locally by a curve $\beta(x, t)=0$. Furthermore, let $u(x, t)$ be a continuous and bounded solution of the heat conduction equation having continuous and bounded derivatives satisfying $u_{x x}=u_{t}$ in the interior of $R$, and let the solution be continuable into the region for which $t>T$. If $u(x, t)$ assumes its maximum or minimum in $R$, say at a point $(\xi, \tau)$, other than at a point of the boundary $\beta(x, t)=0$, then $u$ is a constant in the subregion described as follows: the subregion consists of all points of $R$ which may be reached by a continuous curve $t=f(s), x=g(s)$, where $t=f(s)$ is a monotonic non-increasing function of $s$, starting from any point in $R$ that lies on the line $t=\tau$.

As an engineering application of the Picone theorem, let us consider the following design problem. It is desired to construct a unit for an oven which controls the inside wall temperature of the oven and the heat flux through the wall of the oven, where the oven is heated from the outside, by placing a thermocouple on the outside of the wall of the oven and another on the inside of the wall. We shall assume that the oven is sufficiently large that any cross section of the oven wall may be considered to be part of an infinite slab. Furthermore, we shall assume that the oven receives its heat from a heater distributed uniformly over its entire outside surface. Thus, we shall determine the amount of heat passing through each unit area of the inside of the wall by the temperature drop between the outside and inside of the wall. The temperature distribution $u(x, t)$ across the wall is, in general, given by the solution of the differential equation

$$
\alpha u_{x x}=u_{t}
$$

for the appropriate boundary conditions where $x$ is the space co-

\footnotetext{
Presented to the Society, November 29, 1952; received by the editors December 5 , 1952 and, in revised form, April 27, 1953.

1 Picone, Mauro, Sul problema della propagazione del calore in un mezzo privo di frontiera, conduttore, isotropo e omogeneo, Math. Ann. vol. 101 (1929).

2 Now at University of California Radiation Laboratory, Livermore, California.
} 
ordinate, $t$ is the time, and $\alpha$ is the constant of thermal diffusivity. The steady state solution, obtained by setting $u_{t}=0$ in equation (1), is

$$
u=A x+U
$$

where $k A$ ( $k$ being the constant of thermal conductivity) represents the amount of heat passing through a unit area of the inside of the wall per unit of time. Equation (2) gives the temperature distribution through the wall where $U$ is the inside temperature and $x$ is measured from the inside of the wall outward, the wall thickness being $a$. This choice of coordinate system makes $A$ positive.

Temperature measuring devices of the type described above usually measure a temperature to within a known accuracy, say $\epsilon>0$. Thus, the thermocouple cannot recognize the difference between a temperature $U$ and $U \pm \epsilon$. Similarly, when using two thermocouples, temperature differences, $\Delta U$, cannot be determined with an accuracy greater than a known error $\boldsymbol{\gamma}$. That is, the two thermocouples cannot recognize the difference between $\Delta U$ and $\Delta U \pm \gamma$.

Let us now try to operate the oven as near as possible to the steady state condition in which the inside of the wall is to be maintained at the temperature $U$ with a temperature gradient $A$ through the wall. In order to analyze the operation of the control unit we shall write $\Delta U$ as the difference between the desired temperatures at the outside of the wall and the inside of the wall. Thus,

$$
\Delta U=a A \text {. }
$$

Our control unit will operate as follows:

1) Should

$$
u(0, t) \geqq U+\epsilon
$$

or should

$$
u(0, t)<U+\epsilon, \text { but } \delta U>\Delta U+\gamma
$$

then

$$
u_{x}(a, t)=A-\sigma
$$

where $\sigma>0$ and

$$
\delta U=u(a, t)-u(0, t) .
$$

2) Should

$$
u(0, t) \leqq U-\epsilon
$$


or should

$$
u(0, t)>U-\epsilon \text {, but } \delta U<\Delta U-\gamma
$$

then

$$
u_{x}(a, t)=A+\sigma .
$$

3) neither of the rules 1) or 2) will act twice in succession.

We shall now assume that the oven wall completely encloses the oven and that the object inside being heated always absorbs heat at a constant rate such that

$$
u_{x}(0, t)=A
$$

(for example, a substance undergoing a change of state where such a change is known to occur at constant temperature).

Under the desired steady state operating conditions,

$$
u_{x}(a, t)=A
$$

and the difference between the rate of equation (5) and that of equa(6) is

$$
\Delta u_{x}(a, t)=\sigma .
$$

Similarly, the difference between the rates given by equations (6) and (4) is

$$
\Delta u_{x}(a, t)=\sigma .
$$

Thus, the heat inputs which govern the temperature gradients given by equations (4) and (5) may be made as near as one wishes to the desired input by choosing $\sigma$ sufficiently small.

It is desired to determine if such a controlling device will cause temperature fluctuations which will prohibit its usefulness. To this end we shall show that if the temperature distribution ever lies completely within the $x u$-region, $S$, defined by the straight lines $x=0$, $x=a, u=A x+U+(\epsilon+\gamma)$, and $u=A x+U-(\epsilon+\gamma)$, it will remain within that region. If $u(x, t)$ remains in the region $S$ we see that

$$
\Delta U-2(\epsilon+\gamma) \leqq \delta U \leqq \Delta U+2(\epsilon+\gamma),
$$

that the

$$
\varliminf_{a \rightarrow \infty}\left\{\frac{\delta U}{a}\right\}=\frac{\Delta U}{a}=A,
$$

and that the actual temperature gradient $\delta U / a$ differs from the desired by at most 


$$
\pm \frac{2(\epsilon+\gamma)}{a},
$$

a quantity which may be made as small as we please by increasing the thickness of the oven's wall.

In order to consider the temperature fluctuations initiated by the controlling unit, we must investigate the solutions of two heat conduction problems. The first is the solution of

$$
\alpha u_{x x}=u_{t}
$$

for the initial condition

$$
u(x, 0)=h(x)
$$

where

$$
A x+U-(\epsilon+\gamma) \leqq h(x) \leqq A x+U+(\epsilon+\gamma),
$$

and the boundary conditions

$$
u_{x}(0, t)=A
$$

and

$$
u_{x}(a, t)=A+\sigma .
$$

Let

$$
v(y, t)=u\left(\alpha^{1 / 2} y, t\right)-A \alpha^{1 / 2} y-U+(\epsilon+\gamma)
$$

where $y=x / \alpha^{1 / 2}$. Then $v(y, t)$ satisfies the differential equation,

$$
v_{y y}=v_{t}
$$

of the Picone theorem in the half-open region $R$ of the $y t$-plane defined by the straight lines $y=0, y=a / \alpha^{1 / 2}$, and $t=0 . v(y, t)$ satisfies the initial condition

$$
v(y, 0)=h\left(\alpha^{1 / 2} y\right)-A \alpha^{1 / 2} y-U+(\epsilon+\gamma)
$$

and the boundary conditions

$$
v_{y}(0, t)=0
$$

and

$$
v_{y}\left(a / \alpha^{1 / 2}, t\right)=\alpha^{1 / 2} \sigma .
$$

Since the boundary condition (13) does not allow a constant solution, then, by the Picone theorem, the minimum of $v(y, t)$ must lie on the boundary defined by the lines $y=0, y=a / \alpha^{1 / 2}$, and $t=0$. 
Furthermore, since the boundary conditions (12) and (13) define a heat adding process, the minimum must lie on the boundary $t=0,{ }^{3}$ say at $y=\xi, 0 \leqq \xi \leqq a / \alpha^{1 / 2}$. Thus,

$$
\begin{aligned}
v(y, t) & \geqq v(\xi, 0)=h\left(\alpha^{1 / 2} \xi\right)-A \alpha^{1 / 2} \xi-U+(\epsilon+\gamma) \\
& \geqq A \alpha^{1 / 2} \xi+U-(\epsilon+\gamma)-A \alpha^{1 / 2} \xi-U+(\epsilon+\gamma) \\
& \geqq 0
\end{aligned}
$$

and

$$
u\left(\alpha^{1 / 2} y, t\right)-A \alpha^{1 / 2} y-U+(\epsilon+\gamma) \geqq 0
$$

or

$$
u(x, t) \geqq A x+U-(\epsilon+\gamma) .
$$

Let us re-examine the problem defined by equations (7), (8), (9), and (10), but now let

$$
w(y, t)=u\left(\alpha^{1 / 2} y, t\right)-A \alpha^{1 / 2} y-U-(\epsilon+\gamma) .
$$

$w(y, t)$ also satisfies the differential equation, $w_{v y}=w_{t}$, of the Picone theorem in the half-open region $R$ of the $y t$-plane. $w(y, t)$ satisfies the initial condition

$$
w(y, 0)=h\left(\alpha^{1 / 2} y\right)-A \alpha^{1 / 2} y-U-(\epsilon+\gamma)
$$

and the boundary conditions

$$
w_{y}(0, t)=0
$$

and

$$
w_{y}\left(a / \alpha^{1 / 2}, t\right)=\alpha^{1 / 2} \sigma .
$$

Since the boundary condition (17) does not allow a constant solution, then, by the Picone theorem, the maximum of $w(y, t)$ must occur on the boundary of $R$. Furthermore, the boundary conditions (16) and (17) represent a heat conduction problem in which heat is being added at the surface, $y=a / \alpha^{1 / 2}$, of a slab of thickness $a / \alpha^{1 / 2}$ and the surface of which at $y=0$ is covered by an insulator. Thus, the maxi-

3 That the minimum must lie on the boundary line $t=0$ may also be shown with the aid of the Picone theorem. First, neither the maximum nor minimum can lie on the line $y=0$ for $t>0$. Since $v_{y}(0, t)=0$, the solution may be reflected about the $y=0$ axis; then, by the Picone theorem, since a constant solution is not allowed, the maximum and minimum must lie on one of the boundary lines $y= \pm a / \alpha^{1 / 2}$ or $t=0$. Since the boundary condition on $y=a / \alpha^{1 / 2}$ is $u_{y}\left(a / \alpha^{1 / 2}, t\right)=\alpha^{1 / 2} \sigma$ and that on $y=-a / \alpha^{1 / 2}$ is $u_{y}\left(-a / \alpha^{1 / 2}, t\right)=-\alpha^{1 / 2} \sigma$, a minimum on either of these boundary lines would contradict the boundary conditions there. 
mum must occur either on the line $t=0$ or the line $y=a / \alpha^{1 / 2}$ of the region $R$. Should the maximum occur on $t=0$, say at $y=\eta, 0 \leqq \eta$ $\leqq a / \alpha^{1 / 2}$, then

$$
\begin{aligned}
w(y, t) & \leqq w(\eta, 0)=h\left(\alpha^{1 / 2} \eta\right)-A \alpha^{1 / 2} \eta-U-(\epsilon+\gamma) \\
& \leqq A \alpha^{1 / 2} \eta+U+(\epsilon+\gamma)-A \alpha^{1 / 2} \eta-U-(\epsilon+\gamma) \\
& \leqq 0,
\end{aligned}
$$

and

$$
u\left(\alpha^{1 / 2} y, t\right)-A \alpha^{1 / 2} y-U-(\epsilon+\gamma) \leqq 0
$$

or

$$
u(x, t) \leqq A x+U+(\epsilon+\gamma) .
$$

Should the maximum of $w(y, t)$ occur on $y=a / \alpha^{1 / 2}$, say at $t=\tau>0$, then

$$
w\left(a / \alpha^{1 / 2}, \tau\right) \geqq w(y, t),
$$

for $0 \leqq t \leqq \tau$, and

$$
u(a, \tau) \geqq u(x, t)-A(x-a)
$$

or

$$
u(x, t) \leqq u(a, \tau)+A(x-a)=u(a, \tau)+A x-\Delta U .
$$

Thus, if

$$
u(a, \tau)=U+(\epsilon+\gamma)+\Delta U
$$

then

$$
u(x, t) \leqq A x+U+(\epsilon+\gamma),
$$

which is the result obtained in equation (18). We see from equations (14), (18), and (19) that the solution $u(x, t)$ remains in the $u x$-region $S$ up to and including the time at which the control device (by rule (1)) causes $u_{x}(a, t)$ to switch from the value $A+\sigma$ to the value $A-\sigma$.

Our second heat conduction problem is to investigate the solution of the differential equation (7) for the initial condition (8) and the boundary conditions

$$
u_{x}(0, t)=A
$$

and

$$
u_{x}(a, t)=A-\sigma .
$$

Again, let $w(y, t)=u\left(\alpha^{1 / 2} y, t\right)-A \alpha^{1 / 2} y-U-(\epsilon+\gamma)$. Then, $w(y, t)$ 
satisfies the differential equation of the Picone theorem in the region $R$ for the initial condition

$$
w(y, 0)=h\left(\alpha^{1 / 2} y\right)-A \alpha^{1 / 2} y-U-(\epsilon+\gamma)
$$

and for the boundary conditions

$$
w_{y}(0, t)=0
$$

and

$$
w_{y}\left(a / \alpha^{1 / 2}, t\right)=-\alpha^{1 / 2} \sigma .
$$

Since the boundary condition (23) does not allow a constant solution, by the Picone theorem the maximum of $w(y, t)$ must occur on the boundary of $R$. The boundary conditions (22) and (23) represent a heat conduction problem in which heat is being extracted from one surface of a slab which is insulated on the other surface. Thus, the maximum of $w(y, t)$ must occur on $t=0,{ }^{4}$ say at $y=\xi, 0 \leqq \xi \leqq a / \alpha^{1 / 2}$, and

$$
\begin{aligned}
w(y, t) & \leqq w(\xi, 0)=h\left(\alpha^{1 / 2} \xi\right)-A \alpha^{1 / 2} \xi-U-(\epsilon+\gamma) \\
& \leqq A \alpha^{1 / 2} \xi+U+(\epsilon+\gamma)-A \alpha^{1 / 2} \xi-U-(\epsilon+\gamma) \\
& \leqq 0
\end{aligned}
$$

and

$$
u\left(\alpha^{1 / 2} y, t\right)-A \alpha^{1 / 2} y-U-(\epsilon+\gamma) \leqq 0
$$

or

$$
u(x, t) \leqq A x+U+(\epsilon+\gamma) .
$$

Now, let us re-examine the problem defined by equations (7), (8), (20), and (21), but let

$$
v(y, t)=u\left(\alpha^{1 / 2} y, t\right)-A \alpha^{1 / 2} y-U+(\epsilon+\gamma) .
$$

$v(y, t)$ satisfies the differential equation of the Picone theorem in the region $R$ for the initial condition

$$
v(y, 0)=h\left(\alpha^{1 / 2} y\right)-A \alpha^{1 / 2} y-U+(\epsilon+\gamma)
$$

and for the boundary conditions

$$
v_{y}(0, t)=0
$$

and

- Maximum cannot occur on $y=0$ as shown in footnote 3. Furthermore, the maximum of $w(y, t)$ cannot occur on $y=a / \alpha^{1 / 2}$, since it would contradict the boundary condition (24) there. 


$$
v_{y}\left(a / \alpha^{1 / 2}, t\right)=-\alpha^{1 / 2} \sigma .
$$

Again, this is a heat extraction problem and by the Picone theorem, the minimum of $v(y, t)$ must lie either on the line $t=0$ or the line $y=a / \alpha^{1 / 2}$. Should the minimum of $v(y, t)$ lie on $t=0$, say at $y=\eta$, $0 \leqq \eta \leqq a / \alpha^{1 / 2}$, then

$$
\begin{aligned}
v(y, t) & \geqq v(y, 0)=h\left(\alpha^{1 / 2} \eta\right)-A \alpha^{1 / 2} \eta-U+(\epsilon+\gamma) \\
& \geqq A \alpha^{1 / 2} \eta+U-(\epsilon+\gamma)-A \alpha^{1 / 2} \eta-U+(\epsilon+\gamma) \\
& \geqq 0
\end{aligned}
$$

and

$$
u\left(\alpha^{1 / 2} y, t\right)-A \alpha^{1 / 2} y-U+(\epsilon+\gamma) \geqq 0
$$

or

$$
u(x, t) \geqq A x+U-(\epsilon+\gamma) .
$$

Should the minimum of $v(y, t)$ lie on $y=a / \alpha^{1 / 2}$, say at $t=\tau>0$, then

$$
v\left(a / \alpha^{1 / 2}, \tau\right) \leqq v(y, t)
$$

for $0 \leqq t \leqq \tau$, and

$$
u(a, \tau) \leqq u(x, t)-A(x-a)
$$

or

$$
u(x, t) \geqq u(a, \tau)+A(x-a)=u(a, \tau)+A x-\Delta U
$$

Let

$$
u(a, \tau)=U+\Delta U-(\epsilon+\gamma)
$$

Then

$$
u(x, t) \geqq A x+U-(\epsilon+\gamma),
$$

which is the result obtained in equation (29). Now, we see from equations (25), (29), and (30) that the solution $u(x, t)$ remains in the $u x$-region $S$ up to and including the time at which the control device (by rule (2)) causes $u_{x}(a, t)$ to switch from the value $A-\sigma$ to the value $A+\sigma$.

The author wishes to thank Professor G. C. Evans, University of California, for reading this paper and making suggestions as to content.

Argonne National Laboratory 